OPEN ACCESS

Edited by:

Jose Maria Prieto,

Liverpool John Moores University,

United Kingdom

Reviewed by:

Jwu-Lai Yeh,

Kaohsiung Medical University, Taiwan

Subhalakshmi Ghosh,

Independent Researcher, Kolkata,

India

*Correspondence:

Juntao Feng

jtfeng1976@csu.edu.cn

Biwen Mo

mobiwen2002@sohu.com

${ }^{+}$These authors have contributed equally to this work

Specialty section: This article was submitted to Ethnopharmacology,

a section of the journal

Frontiers in Pharmacology

Received: 17 September 2020

Accepted: 11 January 2021

Published: 22 March 2021

Citation:

Li R, Song P, Tang G, Wei J, Rao L, $M a L$, Jiang $M$, Huang J, $X u Q, W u J$, $L v Q$, Yao $D$, Xiao $B$, Huang $H$, Lei L,

Feng $J$ and Mo B (2021) Osthole Attenuates Macrophage Activation in Experimental Asthma by Inhibitingthe NF-KB/MIF Signaling Pathway. Front. Pharmacol. 12:572463. doi: $10.3389 /$ fphar.2021.572463

\section{Osthole Attenuates Macrophage Activation in Experimental Asthma by Inhibitingthe NF-кB/MIF Signaling Pathway}

\author{
Ruyi $\mathrm{Li}^{1 \dagger}$, Peng Song ${ }^{2,3+}$, Guofang Tang ${ }^{4 \dagger}$, Jianghong $\mathrm{Wei}^{4}$, Lizong Rao ${ }^{4}$, Libing $\mathrm{Ma}^{4}$, \\ Ming Jiang ${ }^{4}$, Jianwei Huang ${ }^{4}$, Qing $\mathrm{Xu}^{4}$, Jingjie $\mathrm{Wu}^{4}$, Qian $\mathrm{Lv}^{4}$, Dong $\mathrm{Yao}^{5}$, Bo Xiao ${ }^{5}$, \\ Haiming Huang ${ }^{5}$, Liping Lei ${ }^{5}$, Juntao Feng ${ }^{1 *}$ and Biwen Mo ${ }^{4 *}$
}

${ }^{1}$ Key Laboratory of National Clinical Research Center for Respiratory Disease, Department of Respiratory and Critical Care Medicine, Xiangya Hospital, Central South University, Changsha, China, ${ }^{2}$ Key Laboratory of Prevention and Treatment for Chronic Diseases by Traditional Chinese Medicine, Affiliated Hospital of Gansu University of Chinese Medicine, Lanzhou, China, ${ }^{3}$ Innovation Research Institute of Traditional Chinese Medicine, Shanghai University of Traditional Chinese Medicine, Shanghai, China, ${ }^{4}$ Department of Respiratory and Critical Care Medicine, Affiliated Hospital of Guilin Medical University, Guilin, China,

${ }^{5}$ Laboratory of Pulmonary Diseases, Guilin Medical University, Guilin, China

Inhibition of activated macrophages is an alternative therapeutic strategy for asthma. We investigated whether a coumarin compound, osthole, isolated from Cnidium monnieri (L.) Cuss, alleviated macrophage activation in vivo and in vitro. Osthole could reduce expression of a marker of activated macrophages, cluster of differentiation (CD)206, in an ovalbumin-challenge model of asthma in mice. Osthole could also inhibit infiltration of inflammatory cells, collagen deposition and production of proinflammatory cytokines [interleukin (IL)-1 $\beta$, tumor necrosis factor- $\bigotimes$, macrophage migration inhibitory factor (MIF)] in asthmatic mice. In vitro, expression of phosphorylated-IKBQ, MIF and M2 cytokines (Ym-1, Fizz-1, arginase-1) in IL-4-induced macrophages decreased upon exposure to the NF-KB inhibitor MG-132. In our short hairpin (sh)RNA-MIFknockdown model, reduced expression of $\mathrm{M} 2$ cytokines was detected in the $\mathrm{IL}-4$ + shRNA-MIF group. Osthole could attenuate the proliferation and migration of an IL4-induced rat alveolar macrophages line (NR8383). Osthole could reduce IL-4induced translocation of nuclear factor-kappa B (NF-kB) in NR8383 cells. Collectively, our results suggest that osthole ameliorates macrophage activation in asthma by suppressing the NF-kB/MIF signaling pathway, and might be a potential agent for treating asthma.

Keywords: osthole, macrophages, asthma, NF-KB, MIF 46

\section{INTRODUCTION}

Asthma is a complex, heterogeneous pulmonary disease that affects $\sim 300$ million people worldwide (Peters et al., 2006; Siracusa et al., 2015). The key features of asthma are infiltration of inflammatory cells, reversible airflow obstruction, mucus secretion and bronchospasm (Medoff et al., 2008). The economic costs of asthma management are high and targeting asthma symptoms in the clinic are limited (Levy et al., 2015). Hence, development of novel, efficacious medications for asthma treatment is important. 


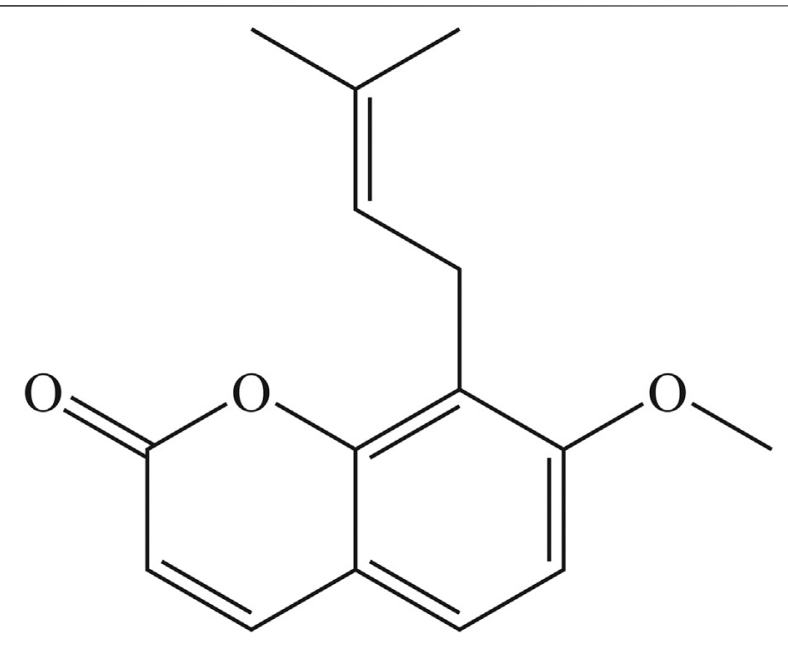

FIGURE 1 | Osthole alleviates OVA-induced macrophage activation and MIF production. (A) Staining (H\&E, Masson, AB-PAS) was undertaken to evaluate infiltration of inflammatory cells, collagen deposition and GC metaplasia ( $\times 200$; scale bar, $100 \mu \mathrm{m}$ ) in lung tissues. (B) The total number of infiltrated cells, lymphocytes and eosinophils in BALF was increased in OVA groups compared with that in the control group. Treatment with osthole $(15,40 \mathrm{mg} / \mathrm{kg})$ or DEX $(1 \mathrm{mg} / \mathrm{kg})$ decreased the numbers of total cells, lymphocytes and eosinophils significantly. (C) Osthole and DEX reduced OVA-induced increased expression of TNF- $\alpha, I L-1 \beta$ and MIF in BALF. (D) mRNA expression of MIF was decreased in asthmatic mice after treatment with osthole or DEX. (E,G) Immunofluorescence for CD206 was conducted to assess accumulation of activated macrophages in lung tissues ( $\times 400$; scale bar, $50 \mu \mathrm{m}$ ). Decreased expression of CD206 was noted in OVA + osthole and OVA + DEX groups compared with that in the OVA group. (F,H) Osthole and DEX decreased OVA-induced increased protein expression of MIF in lung tissues as assessed by immunofluorescence ( $\times 400$; scale bar, $50 \mu \mathrm{m})$. Data are the mean $\pm \mathrm{SD}, \mathrm{n}=5$ mice each group, ${ }^{\star \star \star} p<0.001$ vs. control; $p<$ 0.05 vs. OVA group; $p<0.01$ vs. OVA group; $p<0.001$ vs. OVA group; $\& p<$ 0.05 vs. OVA + Osthole group (15 mg/kg); $p<0.01 v s$. OVA + Osthole group $(15 \mathrm{mg} / \mathrm{kg})$.

Macrophages participate in immune homeostasis in the lung through phagocytosis and chemokine production in response to antigens (Moreira and Hogaboam, 2011). Macrophages (especially M2 macrophages) were discovered originally as a response to the T-helper type 2 (Th2) cytokine interleukin (IL)-4 (Stein et al., 1992). M2 macrophages can be derived from resting macrophages by exposure to IL-4 or IL-13. M2 macrophages are characterized by expression of arginase (Arg)-1, Ym-1, Fizz-1, mannose receptors and scavenger receptors (Gordon, 2003). One of the most prevalent animal asthmatic model used is OVA-sensitized and challenged C57BL/6 mice model, which can effectively induce asthma features, such as airway inflammation, airway smooth muscle mass and goblet cell hyperplasia. These properties are similar to human asthma charicteristics (Kim et al., 2019). Recent studies have shown that depletion of pulmonary alternatively activated macrophages can attenuate airway inflammation in people suffering from asthma (Lee et al., 2015). Therefore, inhibition of activation of Th2-related macrophages may provide an alternative strategy for the clinical treatment of asthma.
Cnidii monnieri Fructus are the dried fruits of Cnidium monnieri (L.) Cusson. C. monnieri Fructus is an important traditional Chinese medicine formulation and used to treat osteoporosis, sexual dysfunction, asthma and skin ailments (Baba et al., 1992). Osthole (also known as "osthol") is found in various plants, including Cnidium monnieri. The pharmacologic activities of osthole include anti-oxidative (Zhang et al., 2011), anti-inflammatory (Liu et al., 2005), antiallergic (Chiu et al., 2008), and anti-diabetes mellitus (Liang et al., 2009) effects. Recent studies have shown that osthole can attenuate the ovalbumin (OVA)-induced inflammation observed in allergic asthma by inhibiting nuclear factor-kappa B (NF-кB) activation (Wang et al., 2017). Data on the role of osthole in macrophage activation in asthma patients are lacking.

Here, we demonstrated that osthole can reduce macrophage activation in OVA-challenged mice and an IL-4-induced macrophage cell line by targeting the NF-кB/macrophage migration inhibitory factor (MIF) pathway.

\section{MATERIALS AND METHODS}

\section{Ethical Approval of the Study Protocol}

The study protocol was approved by the Animal Care and Use Committee of Shanghai University of Traditional Chinese Medicine (PZSHUTCM18120712, Shanghai, China).

\section{Chemicals and Reagents}

Osthole (Figure 1) was purchased from Shanghai Standard Technology (Shanghai, China). OVA (grade V) was obtained from Sigma-Aldrich (Saint Louis, MO, United States). Dexamethasone (Dex) was purchased from Changle Pharmaceuticals (Henan, China). Recombinant murine IL-4 was obtained from Peprotech (Rocky Hill, NJ, United States). MG-132 (NF-кB inhibitor) was purchased from MedChemExpress (Monmouth Junction, NJ, United States). ELISA kits for IL- $1 \beta$, tumor necrosis factor (TNF)- $\otimes$ and MIF for mice were obtained from R\&D Systems (Minneapolis, MN, United States). Western-blot and primary antibodies for immunofluorescence staining were purchased from Cell Signaling Technology (Danvers, MA, United States). All other chemicals were of reagent grade.

\section{Animals}

Specific pathogen-free female C57BL/6 mice (6-8 weeks) were purchased from the Experimental Animals Center of Shanghai University of Traditional Chinese Medicine. Before experimentation, all mice were maintained under standard conditions, and survived on distilled water and standard chow ad libitum, for 7 days.

\section{Sensitization and Challenge With OVA}

We wished to build an asthma model using OVA in mice. Briefly, mice were sensitized by injection (i.p.) with OVA $(100 \mu \mathrm{g})$ and aluminum hydroxide $(2 \mathrm{mg})$ in $200 \mu \mathrm{l}$ of physiologic $(0.9 \%)$ saline on days $0,7,14$ and followed by administration (i.n.) of OVA $(20 \mathrm{mg} / \mathrm{ml})$ in $50 \mu \mathrm{l}$ of $0.9 \%$ saline 
from day-15 to day-28. Non-OVA-challenged mice were sensitized and challenged with $0.9 \%$ saline alone. Mice were divided into five groups of six: control, OVA, OVA + osthole $(15,40 \mathrm{mg} / \mathrm{kg})$, and OVA + Dex (1 mg/kg). Osthole (15, $40 \mathrm{mg} / \mathrm{kg}$ ) was given (i.p.) $1 \mathrm{~h}$ before OVA challenge from day-22 to day-28 for 7 days. Dex ( $1 \mathrm{mg} / \mathrm{kg})$ was given (i.p.) $1 \mathrm{~h}$ before OVA challenge from day-15 to day-28. For the control group, mice were given an equal volume of $0.9 \%$ saline for 14 days. Mice were sacrificed to determine the pathophysiological and immunological features of asthma $24 \mathrm{~h}$ after the final challenge.

\section{Bronchoalveolar Lavage Fluid Collection}

BALF was collected by lavage of the lung twice with $0.5 \mathrm{ml}$ of cold physiologic saline via a tracheal catheter. BALF was centrifuged immediately $\left(2,000 \mathrm{~g}, 5 \mathrm{~min}, 4^{\circ} \mathrm{C}\right)$. The supernatant was used for cytokine measurements. The cell pellet was resuspended in $0.5 \mathrm{ml}$ of phosphate-buffered saline (PBS) and used for total and differential cell counts, which were measured by H\&E-stained cytocentrifuges.

\section{Measurement of Cytokine Levels in BALF and Cell Supernatants}

Concentrations of MIF, IL- $1 \beta$ and TNF- $\alpha$ in BALF and the concentration of MIF protein in supernatants of NR8383 cells were measured by ELISAs according to manufacturer (R\&D Systems) instructions.

\section{Real-Time Reverse Transcription-Quantitative PCR}

Total RNA was isolated from lung tissues and lysates of NR8383 cells by TRIzol ${ }^{\circledR}$ Reagent according to manufacturer (Invitrogen, Carlsbad, CA, United States) instructions. Reverse transcription was carried out with the PrimeScript ${ }^{\circledR}$ RT Reagent Kit (TaKaRa Biotechnology, Shiga, Japan). Next, RT-qPCR was done with the SYBR $^{\circledR}$ Green Premix Ex Taq kit (TaKaRa Biotechnology) on a LightCycler ${ }^{\circledast} 480$ system (Roche, Basel, Switzerland). mRNA expression of target genes was normalized to $\beta$-actin expression in the same sample. The primers we used (forward and reverse, respectively) were: $5^{\prime}$-CCAGAACCGCAACTACAG TAAGC- $3^{\prime}$ and $5^{\prime}$-TTGGCAGCGTTCATGTCGTAATAG- ${ }^{\prime}$ for MIF (mouse); $5^{\prime}$-ATCACTATTGGCAACGAGCGGTTC- $3^{\prime}$ and $5^{\prime}$-CAGCACTGTGTTGGCATAGAGGTC- $3^{\prime}$ for $\beta$-actin (mouse); $5^{\prime}$-TCCGTGCCAGAGGGGTTTCTC- $3^{\prime}$ and $5^{\prime}$-GGG TCGCTCGTGCCACTAAAAG- $3^{\prime}$ for MIF (rat); $5^{\prime}$-CTGAGA GGGAAATCGTGCGTGAC- $3^{\prime}$ and $5^{\prime}$-AGGAAGAGGATG CGGCAGTGG-3’ for $\beta$-actin (rat).

\section{Lung Histopathology}

Lung tissues were fixed with $10 \%$ neutral formalin after mice had been sacrificed. Fixed sections were embedded in paraffin, sectioned and stained with $H \& E$ to examine infiltration of inflammatory cells. Staining [Alcian blue and periodic acidSchiff (AB-PAS), Masson] was used to evaluate mucin-positive goblet cells (GCs) and collagen deposition.

\section{Culture and Transfection of Cells}

The rat alveolar macrophage cell line NR8383 was purchased from Shanghai Zhong Qiao Xin Zhou Biotechnology (Shanghai, China). The peritoneal macrophage cell line RAW264.7 was obtained from Shanghai Institutes for Biological Sciences (Shanghai, China).

Cells were cultured in Dulbecco's modified Eagle's medium (Hyclone, Julich, Germany) supplemented with $10 \%$ fetal bovine serum (Gibco, Grand Island, NY, United States). All cells were grown at $37^{\circ} \mathrm{C}$ in humidified air in an atmosphere of $5 \% \mathrm{CO}_{2}$. Cells were treated with the indicated concentration of osthole according to experimental requirements; the same volume of DMEM was used as the solvent control. A short hairpin RNA (shRNA) sequence targeting MIF in mice was inserted into the PGMLV-hU6-MCS-CMV-ZsGreen1-PGK-Puro-WPRE vector to generate the MIF-si plasmid. The sequence used to silence MIF expression was $5^{\prime}$-GGGTCTACATCAACTATTACG- $3^{\prime}$. The plasmid transfected with scramble siRNA ( $5^{\prime}$-TTCTCC GAACGTGTCACGT-3') was used as a negative control. Lentiviral particles targeting MIF were transfected into RAW264.7 cells. Transfected cells were obtained from Genomeditech (Shanghai, China). The transfection efficiency was confirmed by real-time RT-qPCR (Supplementary Figure S1).

\section{Cell-Viability Assay}

Cell viability was measured using the MTT Cell Viability Assay Kit (Beyotime Biotechnology, Beijing, China) on 96-well plates. After drug treatment, cells were incubated with $20 \mu \mathrm{l}$ of MTT solution $(5 \mathrm{mg} / \mathrm{ml})$ for $4 \mathrm{~h}$ at $37^{\circ} \mathrm{C}$, followed by replacement of the medium with $150 \mu$ of dimethyl sulfoxide. Absorbance was read at $570 \mathrm{~nm}$ on an automated microplate reader (BioTek, Winooski, VT, United States).

\section{Colony-formation Assay}

NR8383 cells were inoculated into 12-well plates at 400 cells/well. Cells were divided into four groups (control, IL-4, IL-4 + osthole, IL- $4+$ Dex) and cultured in an atmosphere of $5 \% \mathrm{CO}_{2}$ at $37^{\circ} \mathrm{C}$ for 14 days. Cells were stained with $0.1 \%$ crystal violet (Beyotime). A digital camera (Nikon, Tokyo, Japan) was used for imaging.

\section{Migration Assay}

Cells were placed into the upper chamber of Transwell ${ }^{\mathrm{TM}}$ inserts (Corning, Corning, NY, United States) with culture medium to detect cell migration. Culture medium was also added to the lower chamber. After $48 \mathrm{~h}$ of incubation at $37^{\circ} \mathrm{C}$, cells migrated through the membrane were fixed with $4 \%$ formaldehyde for $30 \mathrm{~min}$ and stained with $0.1 \%$ crystal violet. Stained cells were imaged and counted under a microscope (Nikon).

\section{Western Blotting}

Cells were treated with NP40 lysis buffer (Beyotime) on ice for $30 \mathrm{~min}$. Lysates were centrifuged at $12,000 \times g$ for $10 \mathrm{~min}$ at $4^{\circ} \mathrm{C}$. The supernatant was collected and protein concentrations were measured with the Protein BCA Assay Kit (Beyotime). Protein samples $\left(30 \mu \mathrm{g}\right.$ of total protein each) were boiled at $100^{\circ} \mathrm{C}$ for $5 \mathrm{~min}$ and separated by sodium dodecyl sulfate-polyacrylamide 


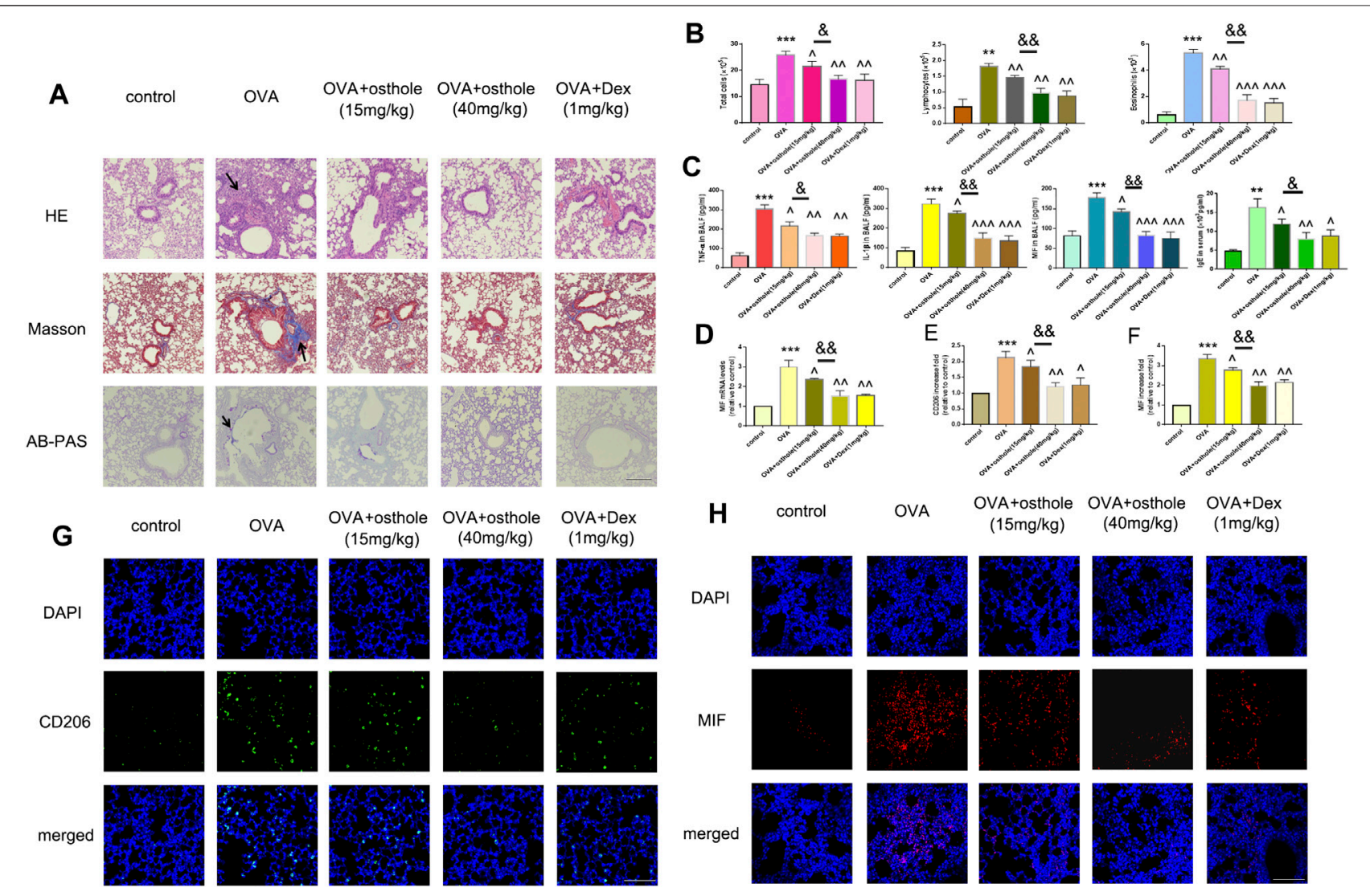

FIGURE 2 | IL-4-induced macrophage activation was mediated by the NF-KB/MIF signaling pathway. (A,B) Phosphorylated and total protein expression of IKB囚 in the presence or absence of NF-KB inhibitor MG-132 was determined by western blotting. (C) MIF secretion in supernatants was measured by ELISA. mRNA expression of MIF in macrophages was measured by RT-qPCR. (D,F) Protein expression of the M2-type cytokines Ym-1, Fizz-1 and Arg-1 was determined by western blotting. (E,G) Protein expression of Ym-1, Fizz-1 and Arg-1 in MIF-knockdown RAW264,7 cells was measured by western blotting. Data are the mean \pm SD. ${ }^{*} p<0.05$ vs. control; ${ }^{\star \star} p<0.01$ vs. control; ${ }^{\star * *} p<0.001$ vs. control; $p<0.05$ vs. IL-4 group; $p<0.01$ vs. IL-4 group.

gel electrophoresis and transferred onto polyvinylidene difluoride (PVDF) membranes (Bio-Rad Laboratories, Hercules, CA, United States). The primary antibodies we used were anti-NFKB-p65 (1:1,000 dilution), anti-MIF (1:1,000), anti-lamin-A (1: $1,000)$, anti-IкBa (1:1,000), anti-phosphorylated (p)-ІкBa (1: 1,000), anti-Ym-1 (1:1,000), anti-Fizz-1 (1:1,000), anti-Arg-1 $(1: 1,000)$ and anti- $\beta$-actin $(1: 1,000)$. This action was followed by incubation with fluorescent-labeled secondary antibodies (1: 5,000; Cell Signaling Technology). Band detection was performed on a platform from Azure Biosystems (Dublin, CA, United States). Quantification of protein levels was done by densitometry using ImageJ v5.0 (National Institutes of Health, Bethesda, MD, United States).

\section{Cell-Proliferation Assay}

Measurement of cell proliferation was based on a colorimetric immunoassay (Abcam, Cambridge, United Kingdom) in which bromodeoxyuridine (BrdU) was incorporated into cells during DNA synthesis. All procedures were undertaken following manufacturer instructions. After preincubation, cells were labeled with BrdU for $2 \mathrm{~h}$. BrdU incorporation was detected by adding an anti-BrdU antibody and the subsequent substrate reaction.

\section{Scratch Assay}

Cells were allowed to reach a confluent monolayer in six-well plates. A conventional pipette was used to make a scratch in the cell monolayer. The monolayer was washed and incubated further. The imaging of scratches was captured by a digital camera system coupled to an inverted microscope (Nikon). Then, cells were incubated for $48 \mathrm{~h}$, and the scratches were imaged by the microscope.

\section{Immunofluorescence}

Sections were fixed using $4 \%$ paraformaldehyde for $24 \mathrm{~h}$ at $4^{\circ} \mathrm{C}$. For immunofluorescence staining of cluster of differentiation (CD)206, MIF and NF-KB-p65, sections were permeabilized with $0.3 \%$ Triton $\mathrm{X}-100$ in PBS for $20 \mathrm{~min}$ at room temperature and blocked with $5 \%$ bovine serum albumin (Beyotime) for $30 \mathrm{~min}$. Then, cells were incubated with primary antibodies overnight at $4^{\circ} \mathrm{C}$. The primary antibodies we used were anti-CD206 (1:350 dilution), anti-MIF (1:300) and anti-NF-кB-p65 (1:200). Subsequently, sections were washed with PBS and then incubated with the appropriate secondary antibody for $2 \mathrm{~h}$ at room temperature. The secondary antibodies we employed were anti-rabbit 


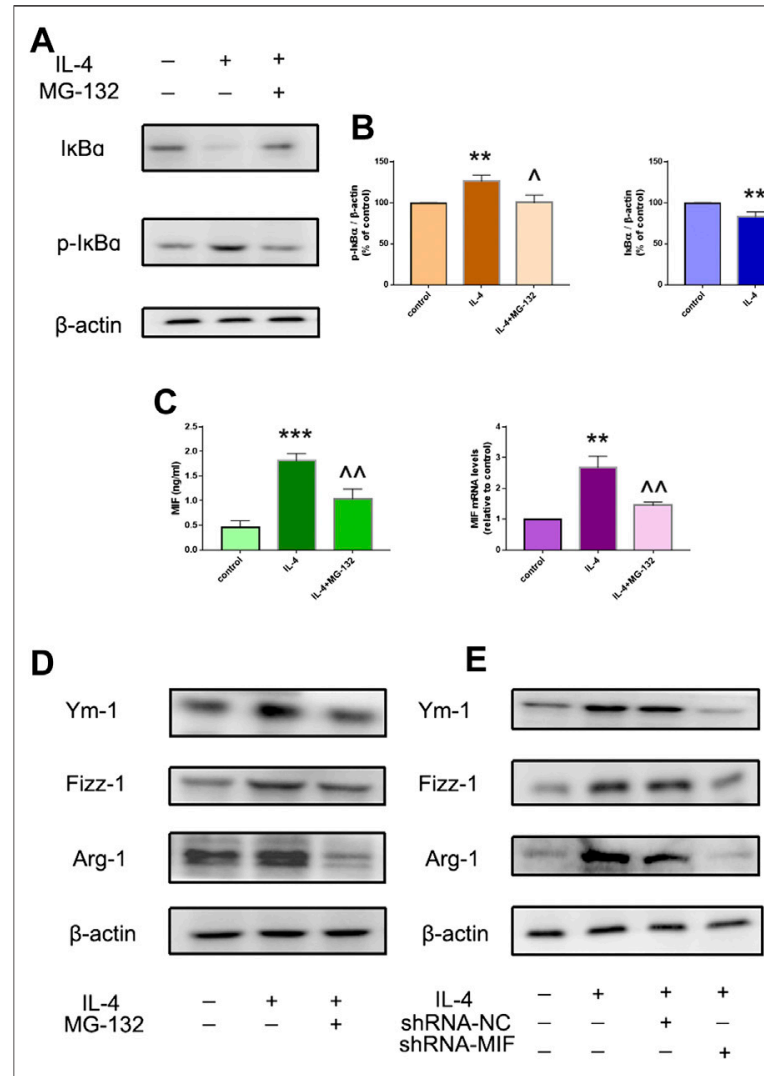

$\mathbf{F}$

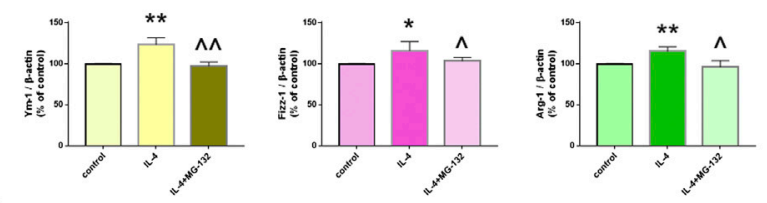

G

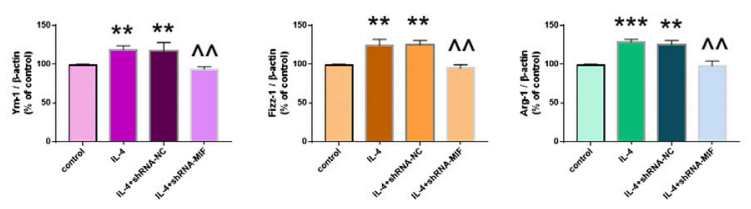

FIGURE 3 | Osthole reduces IL-4-mediated macrophage activation in vitro. (A) Alveolar macrophages were treated with osthole $(0,1,5,10,25$, 50,100 or $200 \mu \mathrm{M}$ ) for $48 \mathrm{~h}$. The toxicity of osthole on macrophages was detected by the MTT assay. (B,C) The colony-formation assay and BrdU cell-proliferation assay were used to analyze cell proliferation. Osthole $(50 \mu \mathrm{M})$ and DEX (100 nM) decreased IL-4-induced macrophage proliferation significantly. (D,E) The scratch assay and Transwell migration assay were used to analyze macrophage migration. Osthole and DEX reduced IL-4induced macrophage migration. Data are the mean \pm SD. ${ }^{* \star} p<0.001$ vs. control; $p<0.001$ vs. IL-4 group.

immunoglobulin (Ig)G (1:450 dilution) and anti-mouse IgG (1: 450), both of which were from Cell Signaling Technology. $4^{\prime}, 6-$ diamidino-2-phenylindole (Invitrogen) staining was used to visualize nuclei. Finally, a laser fluorescence microscope (Nikon) was employed to view immunolabeled slides. Immunofluorescence quantification was carried out using ImageJ v5.0 (National Institutes of Health).

\section{Statistical Analyses}

Data are the mean \pm SD. Differences among groups were assessed by ANOVA and the Student's $t$-test. Statistical analyses were undertaken using Prism 5.0 (GraphPad, San Diego, CA, United States). $\mathrm{P}<0.05$ was considered significant.

\section{RESULTS}

\section{Osthole Inhibited Macrophage Activation and MIF Production in OVA-Challenged Mice}

We wished to determine the role of osthole in an experimental model of asthma. We detected airway remodeling and airway inflammation in an OVA-challenged mice after osthole administration. First, staining (H\&E, Masson, AB-PAS) showed that inflammation infiltration, collagen deposition and GC metaplasia decreased strongly in OVA-challenged asthmatic mice after treatment with osthole (15 and $40 \mathrm{mg} / \mathrm{kg}$ ) and Dex $(1 \mathrm{mg} / \mathrm{kg}$ ) compared with that upon administration of $0.9 \%$ saline (Figure 2A). Second, we counted the number of inflammatory cells (Figure 2B) and protein expression of proinflammatory cytokines in BALF and IgE level in serum (Figure 2C) by ELISA: we documented a decrease in osthole and Dex groups compared with that in the OVA group. With regard to macrophage activation, we counted the number of $\mathrm{CD}_{206}{ }^{+}$cells in lung tissues by fluorescence. The number of $\mathrm{CD}_{206}{ }^{+}$cells was reduced in osthole and Dex groups (Figures 2E,G). Moreover, MIF expression in lung tissues and BALF decreased in asthmatic mice after treatment with osthole or Dex (Figures 2D,F,H). In osthole groups, the decrease in inflammation, macrophage activation and MIF production was in a dose-dependent manner. Airway inflammation, the number of $\mathrm{CD}^{206^{+}}$cells and MIF expression increased in the OVA group compared with that in the control group.

\section{IL-4-Induced Macrophage Activation was Associated With the NF-KB/MIF Signaling Pathway In Vitro}

We wished to assess if IL-4-induced macrophage activation was regulated by NF- $\mathrm{KB} / \mathrm{MIF}$ signaling. NR8383 cells were pretreated with the NF-кB inhibitor MG-132 for $1 \mathrm{~h}$ before stimulation with IL-4 for $24 \mathrm{~h}$. MG-132 suppressed IL-4-related phosphorylation of IкB and MIF secretion greatly (Figures 3A-C). MG-132 could also block IL-4-induced highly expression of Ym-1, Fizz-1 and Arg-1 in NR8383 cells (Figures 3D,F). After exposure to IL-4 for $24 \mathrm{~h}$, MIF-knockdown RAW264.7 cells had reduced expression of Ym-1, Fizz-1 and Arg-1 compared with that in cells transfected with scramble controls (Figures 3E,G).

\section{Osthole Ameliorated Macrophage Activation In Vitro}

As described above, osthole inhibited accumulation of CD206 ${ }^{+}$ cells in a mouse model of asthma. Next, we investigated if osthole 


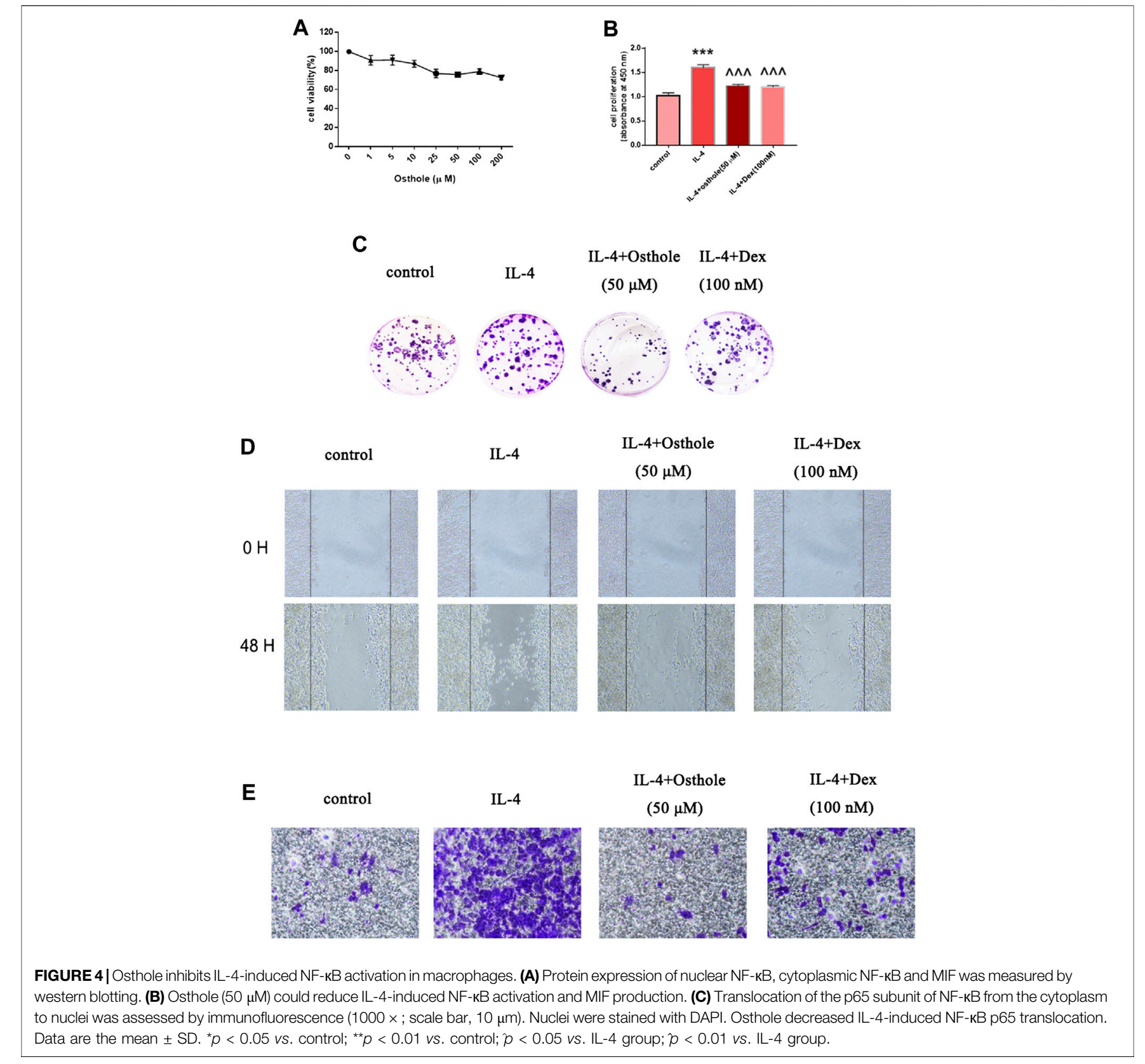

inhibited macrophage activation in vitro. The potential toxicity of osthole to NR8383 cells was measured by the Cell Counting Kit- 8 assay after incubation with osthole for $48 \mathrm{~h}$ (Figure 4A). Osthole did not display toxicity against NR8383 cells. The latter were preincubated with osthole $(50 \mu \mathrm{M})$ for $1 \mathrm{~h}$, then stimulated with IL-4 $(10 \mathrm{ng} / \mathrm{ml})$ for $24 \mathrm{~h}$. Cell proliferation was measured by the BrdU assay (Figure 4B) and colony formation (Figure 4C). Osthole treatment attenuated IL-4-induced macrophage proliferation. The scratch assay (Figure 4D) and Transwell assay (Figure 4E) showed that osthole could reduce IL-4induced macrophage migration. These data suggested that osthole can alleviate IL-4-induced macrophage activation.

\section{Osthole Blocked IL-4-Induced NF-KB Translocation}

We wished to ascertain if osthole could inhibit NF-кB translocation in alveolar macrophages. Cells were pretreated with osthole $(50 \mu \mathrm{M})$ for $1 \mathrm{~h}$ and stimulated with IL-4 (10 ng/ $\mathrm{ml}$ ) for $24 \mathrm{~h}$. Cell lysates were immunoblotted for the p65 subunit of NF-кB. IL-4 stimulated NF-кB translocation significantly, which was prevented effectively by osthole (Figures 5A,B). Immunofluorescence staining of the p65 subunit showed that osthole pretreatment prevented the IL-4-induced nuclear translocation of NF-кB (Figure 5C). Western blotting showed the inhibitive effect of osthole on IL-4-induced MIF expression 


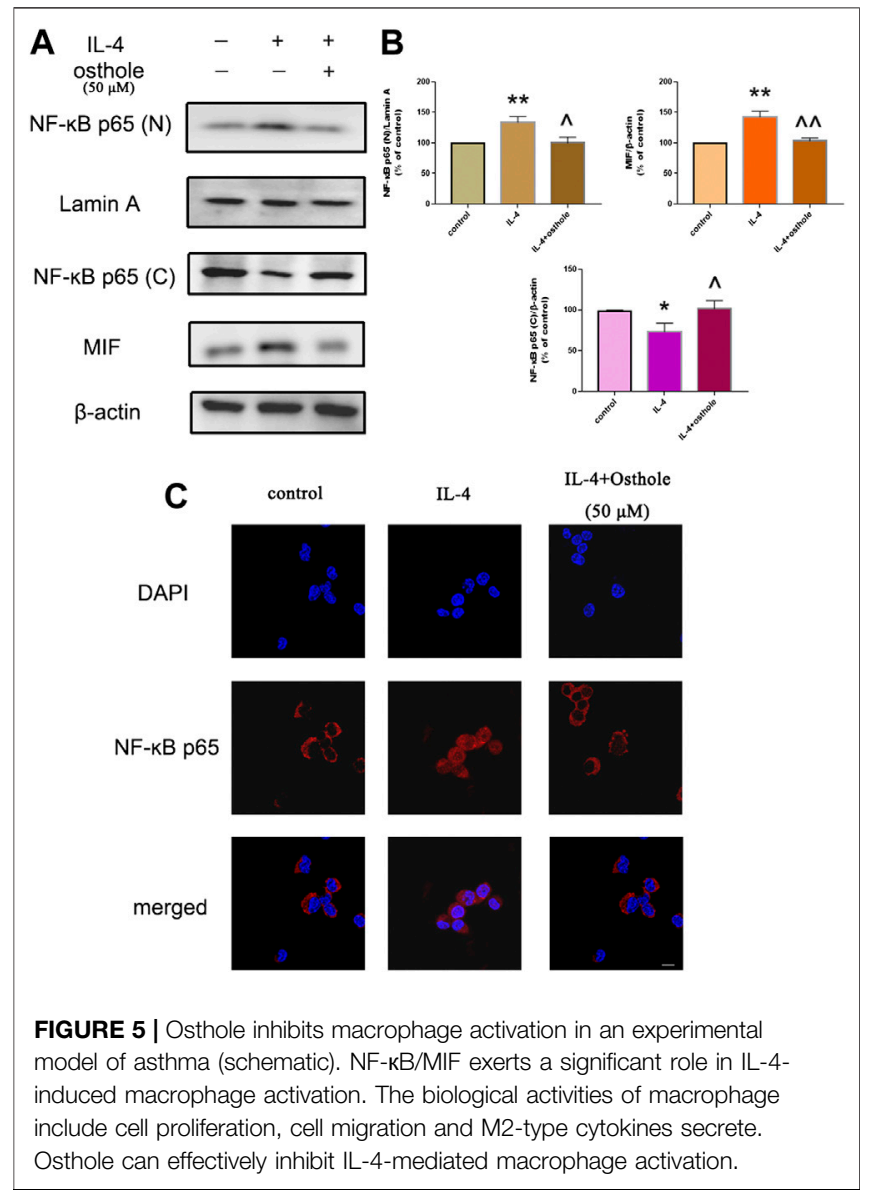

(Figures 5A,B). These data suggested that osthole suppressed IL4 -induced NF-кB activation.

\section{DISCUSSION}

The present study elicited two novel findings: i) osthole can ameliorate airway inflammation and macrophage activation in a murine model of asthma; ii) the inhibitive effect of osthole on macrophage activation may be associated with a NF-KB/MIF dependent signaling pathway.

Osthole can inhibit immune inflammatory diseases such as arthritis and hepatitis (Okamoto et al., 2001; Okamoto et al., 2003). In a model of alcoholic fatty liver disease, osthole led to decreased oxidative stress and increased activation of superoxide dismutase (Zhang et al., 2011). Studies have shown that osthole attenuated lipopolysaccharide-induced acute lung injury in a murine model (Chen et al., 2013; Shi et al., 2013). Previous report indicated that the treatment with coumarins was able to cause drowsiness and stomach discomfort in asthma patients. It has been found that the maximum tolerated dose of osthole in mice was $1.50 \mathrm{~g} / \mathrm{kg}$, which showed osthole had a limited toxicity (Yang and Liu, 2012). Osthole can attenuate OVA-induced inflammation in allergic asthma by inhibiting NF-кB activation, but whether osthole can regulate macrophage activation in asthma (and the specific mechanism of action) is not known.

We investigated the effect of osthole on macrophage activation in OVA-induced asthma. First, we measured expression of airway inflammation-associated markers in asthmatic mice. Expression of IL- $1 \beta$ and TNF- $\alpha$ in BALF was reduced after osthole exposure. Histology also revealed inflammation and remodeling to be reduced in mice exposed to osthole. CD206 is one of the most important markers of activated M2 macrophages, so we detected the immunofluorescence of CD206 in lung tissues. We discovered that reduced expression of CD206 was involved in our OVAchallenged model of asthma after osthole treatment. We showed that osthole inhibited airway inflammation, airway remodeling and macrophage activation in our asthma model, and MIF expression was also suppressed in the osthole group. These findings suggest that osthole might be a potential anti-allergic agent for asthma treatment.

Recent studies have demonstrated that macrophages have pivotal roles in the modulation of cancer, type-II diabetes mellitus, cystic fibrosis and atherosclerosis (Sica and Mantovani, 2012; Wynn et al., 2013). Traditionally, lymphocytes, B cells, mast cells and dendritic cells are considered to be associated with a type2 immune response, but activated M2 macrophages also contribute to the inflammation seen in asthma (Sica and Mantovani, 2012; Qian

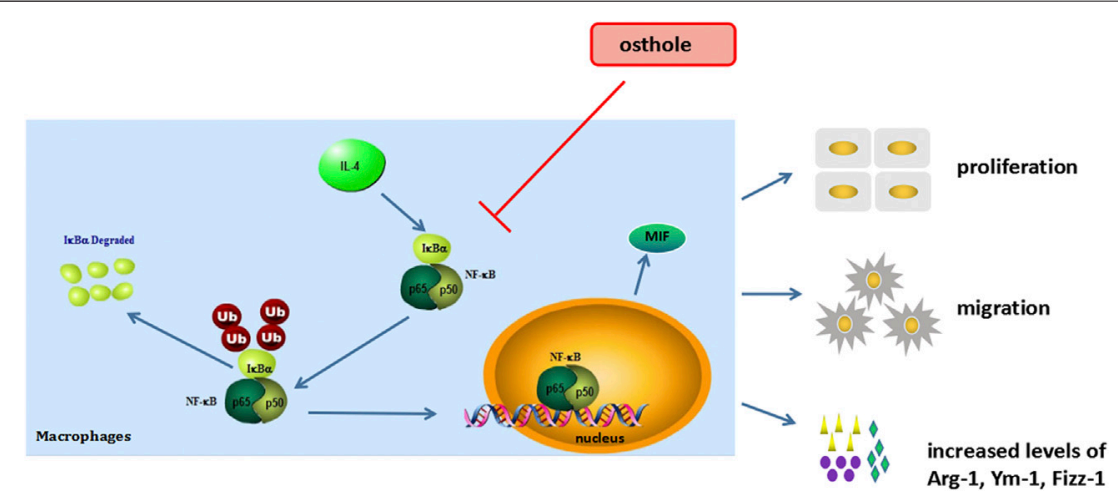

FIGURE 6 | Osthole inhibits macrophage activation in an experimental model of asthma (schematic). NF- $\mathrm{kB} / \mathrm{MIF}$ exerts a significant role in IL-4-induced macrophage activation. The biological activities of macrophage include cell proliferation, cell migration and M2-type cytokines secrete. Osthole can effectively inhibit IL-4mediated macrophage activation. 
et al., 2015). Wang and colleagues demonstrated that osthole attenuated the development of pancreatic cancer in mice by inhibiting tumor-infiltrating M2 macrophages (Wang et al., 2018). We explored the role of osthole in IL-4-induced macrophage activation in vitro. Colony-formation and BrdU cellproliferation assays demonstrated that osthole could suppress IL-4induced macrophage proliferation. Scratch and Transwell assays also revealed that osthole inhibited IL-4-induced macrophage migration.

The transcriptional factor NF- $\mathrm{KB}$ has a pivotal role in the recruitment of inflammatory cells and production of Th2 cytokines in murine models of asthma (Choi et al., 2004; Kang et al., 2009). The NF-KB/MIF signaling pathway can regulate fatty acid-induced dysfunction and apoptosis of $\beta$-cells in pancreatic islets (Zheng et al., 2017). In the acute lung injury model, the inhibitory effect of osthole on eukocytic recruitment, and cytokine productions was regulated by NF-кB (Jin et al., 2018). Osthole was also proved to alleviate pulmonary arterial hypertension in monocrotaline-induced rats, which was closely associated with NF-кB pathway (Li et al., 2017). Osthole exerts an anti-inflammation effect in asthma by inhibiting NF-кB activation (Wang et al., 2017). We showed that production of IL4-induced M2-type cytokines in macrophages was closely associated with the NF-кB/MIF signaling pathway. Osthole inhibited NF-кB activation, thereby suppressing IL-4-induced translocation of transcription factors into nuclei. These findings suggest that osthole-mediated macrophage activation in asthma may be associated with inhibition of the NF-KB/MIF signaling pathway, which may explain the therapeutic effects of osthole.

\section{CONCLUSION}

We demonstrated that osthole inhibited airway inflammation and macrophage activation in a mouse model of OVA-induced asthma. Osthole could suppress IL-4-induced macrophage activation, including cell proliferation, cell migration and production of M2 cytokines. The inhibitive effects of osthole in an experimental model of asthma may be mediated by the NF-KB/MIF signaling pathway (Figure 6). Our findings support the potential application of osthole as a therapeutic drug against allergic asthma.

\section{REFERENCES}

Baba, K., Kawanishi, H., Taniguchi, M., and Kozawa, M. (1992). Chromones from Cnidium monnieri. Phytochemistry 31 (4), 1367-1370. doi:10.1016/00319422(92)80292-m

Chen, X. J., Zhang, B., Hou, S. J., Shi, Y., Xu, D. Q., Wang, Y. X., et al. (2013). Osthole improves acute lung injury in mice by up-regulating Nrf-2/thioredoxin 1. Respir. Physiol. Neurobiol. 188, 214-222. doi:10.1016/j.resp.2013.04.014

Chiu, P. R., Lee, W. T., Chu, Y. T., Lee, M. S., Jong, Y. J., and Hung, C. H. (2008). Effect of the chinese herb extract osthol on IL-4-induced eotaxin expression in BEAS-2B cells. Pediatr. Neonatol. 49, 135-140. doi:10.1016/S1875-9572(08)60028-5

Choi, I. W., Kim, D. K., Ko, H. M., and Lee, H. K. (2004). Administration of antisense phosphorothioate oligonucleotide to the p65 subunit of NF-kappaB inhibits established asthmatic reaction in mice. Int. Immunophar. 4, 1817-1828. doi:10.1016/j.intimp.2004.07.030

Gordon, S. (2003). Alternative activation of macrophages. Nat. Rev. Immunol. 3, 23-35. doi:10.1038/nri978

\section{DATA AVAILABILITY STATEMENT}

The raw data supporting the conclusions of this article will be made available by the authors, without undue reservation.

\section{ETHICS STATEMENT}

The animal study was reviewed and approved by the Institutional Animal Care and Use Committee of Shanghai University of Traditional Chinese Medicine.

\section{AUTHOR CONTRIBUTIONS}

BM and JF designed the study and drafted the manuscript. RL, PS and GT carried out the experiments and drafted the manuscript. RL, JWe, LR, LM, MJ, JH, QX, JWu, and QL participated in data analyses. PS, DY, BX, HH, and LL were involved in discussions regarding the experiments. All authors approved the final version of the manuscript for submission.

\section{FUNDING}

The present study was supported by the National Natural Science Foundation of China $(81960007,81460005,81760008$, and 81803779), Guangxi Natural Science Foundation Program (2015GXNSFAA139107) and the High Level of Innovation Team and Outstanding Scholars Program in Colleges and Universities in Guangxi. Gansu Province Science Foundation for Distinguished Young Scholars (Grant No. 20JR10RA348).

\section{SUPPLEMENTARY MATERIAL}

The Supplementary Material for this article can be found online at: https://www.frontiersin.org/articles/10.3389/fphar.2021.572463/ full\#supplementary-material.

FIGURE S1 | RT-qPCR suggested that MIF expression was blocked effectively in shRNA-MIF510 transfected RAW264.7 cells. ${ }^{*} p<0.05$ vs. control.

Jin, Y., Qian, J., Ju, X., Bao, X., Li, L., Zheng, S., et al. (2018). Osthole protects against acute lung injury by suppressing NF-kB-dependent inflammation. Meditors Inflamm. 2018, 4934592. doi:10.1155/2018/4934592

Kang, N. I., Yoon, H. Y., Lee, Y. R., Won, M., Chung, M. J., Park, J. W., et al. (2009). A20 attenuates allergic airway inflammation in mice. J. Immunol. 183, 1488-1495. doi:10.4049/jimmunol.0900163

Kim, D. I., Song, M. K., and Lee, K. (2019). Comparison of asthma phenotypes in OVA-induced mice challenged via inhaled and intranasal routes. BMC Pulm. Med. 19, 241. doi:10.1186/s12890-019-1001-9

Lee, Y. G., Jeong, J. J., Nyenhuis, S., Berdyshev, E., Chung, S., Ranjan, R., et al. (2015). Recruited alveolar macrophages, in response to airway epithelialderived monocyte chemoattractant protein $1 / \mathrm{CCl} 2$, regulate airway inflammation and remodeling in allergic asthma. Am. J. Respir. Cell Mol. Biol. 52, 772-784. doi:10.1165/rcmb.2014-0255OC

Levy, B. D., Noel, P. J., Freemer, M. M., Cloutier, M. M., Georas, S. N., Jarjour, N. N., et al. (2015). Future research directions in asthma. an NHLBI working group report. Am. J. Respir. Crit. Care Med. 192, 1366-1372. doi:10.1164/rccm. 201505-0963WS 
Li, Y., Wang, Y., Li, Y., Qian, Z., Zhu, L., and Yang, D. (2017). Osthole attenuates pulmonary arterial hypertension in monocrotaline-treated rats. Mol. Med. Rep. 16, 2823-2829. doi:10.3892/mmr.2017.6876

Liang, H. J., Suk, F. M., Wang, C. K., Hung, L. F., Liu, D. Z., Chen, N. Q., et al. (2009). Osthole, a potential antidiabetic agent, alleviates hyperglycemia in $\mathrm{db} /$ db mice. Chem. Biol. Interact 181, 309-315. doi:10.1016/j.cbi.2009.08.003

Liu, J., Zhang, W., Zhou, L., Wang, X., and Lian, Q. (2005). Anti-inflammatory effect and mechanism of osthole in rats. Zhong Yao Cai 28, 1002-1006. doi:10. 13863/j.issn1001-4454.2005.11.016

Medoff, B. D., Thomas, S. Y., and Luster, A. D. (2008). T cell trafficking in allergic asthma: the ins and outs. Annu. Rev. Immunol. 26, 205-232. doi:10.1146/ annurev.immunol.26.021607.090312

Moreira, A. P., and Hogaboam, C. M. (2011). Macrophages in allergic asthma: finetuning their pro- and anti-inflammatory actions for disease resolution. J. Interfe. Cytoki. Res. 31, 485-491. doi:10.1089/jir.2011.0027

Okamoto, T., Kawasaki, T., and Hino, O. (2003). Osthole prevents anti-Fas antibodyinduced hepatitis in mice by affecting the caspase-3-mediated apoptotic pathway. Biochem. Pharmacol. 65, 677-681. doi:10.1016/s0006-2952(02)01606-4

Okamoto, T., Yoshida, S., Kobayashi, T., and Okabe, S. (2001). Inhibition of concanavalin A-induced mice hepatitis by coumarin derivatives. Jpn. J. Pharmacol. 85, 95-97. doi:10.1254/jjp.85.95

Peters, S. P., Ferguson, G., Deniz, Y., and Reisner, C. (2006). Uncontrolled asthma: a review of the prevalence, disease burden and options for treatment. Respir. Med. 100, 1139-1151. doi:10.1016/j.rmed.2006.03.031

Qian, F., Deng, J., Lee, Y. G., Zhu, J., Karpurapu, M., Chung, S., et al. (2015). The transcription factor PU.1 promotes alternative macrophage polarization and asthmatic airway inflammation. J. Mol. Cell Biol. 7, 557-567. doi:10.1093/jmcb/mjv042

Shi, Y., Zhang, B., Chen, X. J., Xu, D. Q., Wang, Y. X., Dong, H. Y., et al. (2013). Osthole protects lipopolysaccharide-induced acute lung injury in mice by preventing down-regulation of angiotensin-converting enzyme 2. Eur. J. Pharm. Sci. 48, 819-824. doi:10.1016/j.ejps.2012.12.031

Sica, A., and Mantovani, A. (2012). Macrophage plasticity and polarization: in vivo veritas. J. Clin. Invest. 122, 787-795. doi:10.1172/JCI59643

Siracusa, C. M., Brewington, J. J., Brockbank, J. C., and Guilbert, T. W. (2015). Update in pediatric lung disease 2014. Am. J. Respir. Crit. Care Med. 192, 918-923. doi:10.1164/rccm.201504-0752UP
Stein, M., Keshav, S., Harris, N., and Gordon, S. (1992). Interleukin 4 potently enhances murine macrophage mannose receptor activity: a marker of alternative immunologic macrophage activation. J. Exp. Med. 176, 287-292. doi:10.1084/jem.176.1.287

Wang, B., Zheng, X., Liu, J., Zhang, Z., Qiu, C., Yang, L., et al. (2018). Osthole inhibits pancreatic cancer progression by directly exerting negative effects on cancer cells and attenuating tumor-infiltrating M2 macrophages. J. Pharmacol. Sci. 137, 290-298. doi:10.1016/j.jphs.2018.07.007

Wang, J., Fu, Y., Wei, Z., He, X., Shi, M., Kou, J., et al. (2017). Anti-asthmatic activity of osthole in an ovalbumin-induced asthma murine model. Respir. Physiol. Neurobiol. 239, 64-69. doi:10.1016/j.resp.2017.01.011

Wynn, T. A., Chawla, A., and Pollard, J. W. (2013). Macrophage biology in development, homeostasis and disease. Nature 496, 445-455. doi:10.1038/ nature 12034

Yang, X. G., and Liu, X. L. (2012). Study of osthol acute toxicity in mice. Chin. Anim. Husb. Vet. Dig. 28, 196-198.

Zhang, J., Xue, J., Wang, H., Zhang, Y., and Xie, M. (2011). Osthole improves alcohol-induced fatty liver in mice by reduction of hepatic oxidative stress. Phytother Res. 25, 638-643. doi:10.1002/ptr.3315

Zheng, S., Ren, X., Han, T., Chen, Y., Qiu, H., Liu, W., et al. (2017). Fenofibrate attenuates fatty acid-induced islet $\beta$-cell dysfunction and apoptosis via inhibiting the NF- $\mathrm{B} / \mathrm{MIF}$ dependent inflammatory pathway. Metab. Clin. Exp. 77, 23-38. doi:10.1016/j.metabol.2017. 09.001

Conflict of Interest: The authors declare that the research was conducted in the absence of any commercial or financial relationships that could be construed as a potential conflict of interest.

Copyright (c) $2021 \mathrm{Li}$, Song, Tang, Wei, Rao, Ma, Jiang, Huang, Xu, Wu, Lv, Yao, Xiao, Huang, Lei, Feng and Mo. This is an open-access article distributed under the terms of the Creative Commons Attribution License (CC BY). The use, distribution or reproduction in other forums is permitted, provided the original author(s) and the copyright owner(s) are credited and that the original publication in this journal is cited, in accordance with accepted academic practice. No use, distribution or reproduction is permitted which does not comply with these terms. 